\title{
Cirugía de las metástasis suprarrenales
}

\author{
Surgery of the adrenal metastases
}

Helga K. Kälviäinen-Mejía*, Pablo Sancho-Pardo, José M. Miguelena-Bobadilla, M. Carmen Casamayor-Franco y Miguel A. Dobón-Rascón

Hospital Universitario Miguel Servet, Zaragoza, España

\section{Resumen}

Antecedentes: Las metástasis suprarrenales son las lesiones malignas más frecuentes de las glándulas suprarrenales. Como localización del tumor primario se encuentran el pulmón (39\%), la mama (35\%), el riñón y el tracto gastrointestinal, entre otros. La cirugía mejora la supervivencia en casos seleccionados. Método: Estudio observacional, retrospectivo y unicéntrico de pacientes intervenidos de suprarrenalectomía por metástasis suprarrenal en un periodo de 11 años. Se describen las características de la enfermedad y los resultados quirúrgicos. Resultados: Se realizaron 14 suprarrenalectomías. La media de edad de los pacientes fue de 65.85 años. Los tumores primarios fueron carcinoma no microcítico pulmonar (42.8\%) y carcinoma renal de células claras (14.20\%). En el 92.8\% de los casos fue unilateral y en el 64.2\% metacrónica. Se realizó laparoscopia inicial en el 85.71\%. La morbilidad fue del 14.28\%. La mediana de supervivencia global fue de 30 meses. La supervivencia fue del $75 \%$ al año, el 55.5\% a los 3 años y el 40\% a los 5 años. Conclusiones: La edad, la localización primaria, el grado de diferenciación, el tipo histológico, el tamaño, la lateralidad, el intervalo libre de enfermedad, la quimioterapia y la técnica quirúrgica no se asocian a cambios en la supervivencia. Ante una masa suprarrenal única, la valoración quirúrgica es mandatoria y la cirugía podría ocupar un papel en pacientes con metástasis en otras localizaciones con control de la enfermedad primaria.

Palabras clave: Metástasis suprarrenal. Factores asociados. Suprarrenalectomía. Supervivencia.

\begin{abstract}
Background: Adrenal metastases are the most common malignant lesions of the adrenal glands and the second most common tumor after adenomas. The location of the primary tumor is described: lung (39\%), breast (35\%), gastrointestinal tract, among other. Several studies show that surgery improves survival in selected cases. Method: Retrospective and single-center observational study of patients operated for adrenal metastasis over a period of 11 years. The characteristics of the disease and surgical results were described. Results: 14 suprarenalectomies were performed. The average age was 65.85 years. The primary tumors described: non-small cell lung carcinoma (42.8\%) and clear cell renal carcinoma (14.20\%). In 92.8\% the injury was unilateral. In $64.2 \%$ it was metachronous. An initial laparoscopic approach was performed in $85.71 \%$. The morbidity of our series was $14.28 \%$. The median overall survival was 30 months. Survival was $75 \%$ per year, $55.5 \%$ at 3 years and $40 \%$ at 5 years. Conclusions: Age, primary location, degree of differentiation, histological type, size greater, laterality, disease-free interval, chemotherapy and surgical technique are not associated with changes in survival. In the presence of a single adrenal mass, surgical evaluation is mandatory and surgery could play a role in patients with metastases in other locations with control of the primary disease.
\end{abstract}

Key words: Adrenal metastasis. Associated factors. Adrenalectomy. Survival

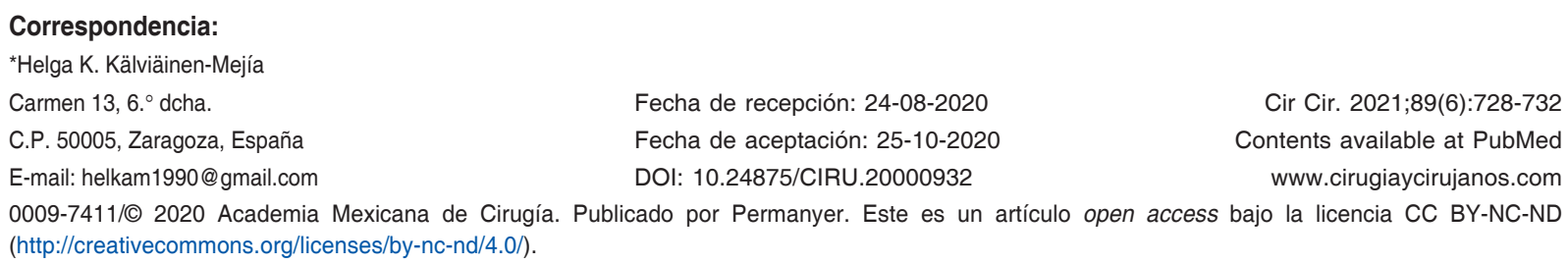

Fecha de recepción: 24-08-2020

Fecha de aceptación: 25-10-2020

DOI: 10.24875/CIRU.20000932

Cir Cir. 2021;89(6):728-732

Contents available at PubMed www.cirugiaycirujanos.com 


\section{Introducción}

Las metástasis suprarrenales son las lesiones malignas más frecuentes de las glándulas suprarrenales y el segundo tumor más común después de los adenomas. Actualmente, con el uso de pruebas de imagen para el diagnóstico, la estadificación y el seguimiento de tumores malignos, son diagnosticadas cada vez más de manera incidental.

Las glándulas suprarrenales son el cuarto sitio más habitual de metástasis, y alrededor del $30 \%$ al $70 \%$ de las masas descubiertas incidentalmente en pacientes con antecedentes de cáncer o de neoplasia extrarrenal suprarrenal recientemente diagnosticada son metástasis ${ }^{1,2}$.

Se describen como localización del tumor primario el pulmón (39\%), la mama (35\%), el melanoma, el tracto gastrointestinal, el páncreas y el riñón, entre otros lugares ${ }^{3}$.

La diseminación metastásica suprarrenal se produce por vía hematógena, y puede ser bilateral debido a su importante irrigación ${ }^{4}$. Se describen de forma aislada en menos del $1 \%$ de los casos ${ }^{5}$.

La mayoría de los pacientes con metástasis suprarrenal son asintomáticos, aunque pueden tener síntomas localizados, como dolor de espalda o abdominal debido al efecto de masa tumoral y a la posible afectación de los nervios esplácnicos. No son tumores «funcionantes» ni suelen provocar insuficiencia suprarrenal; la afectación suprarrenal bilaterales inferior al $90 \%$.

La mediana del tiempo desde el diagnóstico de cáncer hasta la identificación de metástasis suprarrenales es de aproximadamente 2.5 años, aunque se han descrito hasta 22 años después del tratamiento inicial de los tumores primarios. Es poco frecuente que ocurran metástasis suprarrenales aisladas antes de la identificación de la neoplasia maligna primaria ${ }^{6}$.

La cirugía desempeña un papel importante en el tratamiento de las lesiones suprarrenales sospechosas de metástasis, pues diversos estudios describen una mejora en la supervivencia tras la resección.

\section{Método}

Se realizó un estudio observacional retrospectivo y unicéntrico que incluyó a todos los pacientes mayores de 18 años que con diagnóstico de metástasis suprarrenales fueron intervenidos de suprarrenalectomía en la unidad de cirugía endocrina de un hospital de tercer nivel, con un informe favorable del comité de tumores endocrinos del centro, en un periodo de 11 años (2009-2019). Los datos se obtuvieron de la historia clínica electrónica del hospital.

Las metástasis fueron consideradas como sincrónicas si se detectaron en un plazo inferior a 6 meses desde el diagnóstico del tumor primario, y como metacrónicas si el intervalo era mayor. Se decidió utilizar esta definición de acuerdo con la literatura revisada, en la que también se ha publicado algún caso con la denominación de metastasectomía antecrónica?.

Se describieron las características demográficas, la comorbilidad, los antecedentes médico-quirúrgicos, la presentación clínica, las características de la cirugía, los resultados quirúrgicos, la anatomía patológica, la mortalidad y la supervivencia.

En la presentación clínica de la enfermedad se describió la presencia de síntomas o de insuficiencia suprarrenal en su caso.

Las cirugías fueron realizadas por especialistas de la unidad de cirugía endocrina. Se describieron el tipo de cirugía, el abordaje y el tiempo quirúrgico.

La mortalidad perioperatoria se definió como la ocurrida durante el ingreso o bien dentro de los 30 días del posoperatorio.

El análisis estadístico se realizó con el Statistical Package for the Social Sciences (SPSS v. 15). Las variables cuantitativas con distribución normal según la prueba de Kolmogorov-Smirnov se compararon usando la prueba t de Student, en tanto que en evidencia de no normalidad se empleó la prueba de Mann-Whitney. Las variables categóricas se compararon mediante la prueba de $\chi^{2}$ (test exacto de Fisher). Se consideró un valor $p<0.05$ como significativo.

\section{Resultados}

En nuestro centro se realizaron 113 suprarrenalectomías en el periodo de estudio (2009-2019), de las cuales $14(12.4 \%)$ fueron por metástasis suprarrenales. El $71.40 \%$ ( 10 casos) eran hombres y el $28.57 \%$ (4 casos) eran mujeres, con una media de edad de 65.85 años.

Los tumores primarios más frecuentes fueron carcinoma no microcítico pulmonar (42.8\%) y carcinoma renal de células claras (14.20\%), encontrando también metástasis de sarcoma fusocelular, carcinoma lobulillar infiltrante de mama, hepatocarcinoma, adenocarcinoma colorrectal y adenocarcinoma esofágico (Tabla 1). 
Tabla 1. Lesiones primarias causantes de metástasis en nuestra serie

\begin{tabular}{lcc}
\hline Tumor primario & Casos & Porcentaje \\
\hline Carcinoma no microcítico pulmonar & 6 & $42.8 \%$ \\
Carcinoma de células claras renal & 2 & $14.20 \%$ \\
Carcinoma neuroendocrino pulmonar & 1 & $7.14 \%$ \\
Sarcoma fusocelular & 1 & $7.14 \%$ \\
Carcinoma lobulillar infiltrante de mama & 1 & $7.14 \%$ \\
Hepatocarcinoma & 1 & $7.14 \%$ \\
Adenocarcinoma colorrectal & 1 & $7.14 \%$ \\
Adenocarcinoma de esófago & 1 & $7.14 \%$ \\
\hline
\end{tabular}

En el $92.8 \%$ (13 casos) la lesión fue unilateral. En caso de afectación bilateral, el tumor primario se localizaba en el pulmón. La suprarrenal más afectada fue la del lado derecho (57.1\%), y en los casos de tumor renal de células claras, en un $50 \%$ la metástasis era homolateral.

En el $64.2 \%$ (9 casos), la metástasis suprarrenal era metacrónica, con una media de tiempo de aparición desde el diagnóstico primario de 48 meses (8-132 meses). El $28.57 \%$ (4 casos) eran metástasis sincrónicas y hubo un caso de metástasis antecrónica.

En 13 pacientes se diagnosticó y confirmó la lesión metastásica con tomografía computarizada (TC) y tomografía por emisión de positrones (PET); solo en un caso no se realizó PET/TC, y en un caso de carcinoma no microcítico pulmonar se realizó punción-aspiración con aguja fina.

El diagnóstico de metástasis suprarrenales en todos los casos fue un hallazgo. Así mismo, al diagnóstico, seis pacientes $(48,8 \%)$ tenían lesiones metastásicas en otra localización y siete pacientes recibieron neoadyuvancia. En el 85.7\% (12 casos) se realizó la cirugía del tumor primario antes que la suprarrenalectomía. En un caso, el tumor primario fue diagnosticado posterior a la cirugía suprarrenal, y en el otro se desestimó la cirugía del primario por tratarse de un carcinoma escamoso de esófago en estadio IV.

En cuanto a las características quirúrgicas de nuestra serie, se realizó un abordaje laparoscópico inicial en el $85.71 \%$ (12 casos), convirtiendo a cirugía abierta solo un caso debido a sangrado abundante. En el resto de los pacientes se decidió cirugía abierta inicial por tratarse de tumores con un tamaño $>14 \mathrm{~cm}$. En dos casos se asoció resección de otros órganos, en uno de ellos gastrectomía subtotal y en otro segmentectomía hepática del $\mathrm{V}$ segmento. El tiempo medio de la cirugía fue de 241 minutos. El $28.5 \%$ (4 casos) requirieron transfusión sanguínea.

La media de tamaño tumoral fue de $5.33 \mathrm{~cm}$ (1.3$15 \mathrm{~cm}$ ), y el grado histológico fue G1 en el 7.1\%, G2 en el $42.85 \%$ y G3 en el $35.7 \%$. Se realizó una resección completa en el $85.71 \%$ (12 casos).

La morbilidad de nuestra serie fue del $14.28 \%$ : dos casos clasificados como Clavien-Dindo IIla y V, respectivamente. Un paciente presentó fístula biliar posoperatoria (el caso de segmentectomía hepática asociada) que requirió drenaje percutáneo y otro sufrió shock hemorrágico con resultado de muerte. La mortalidad posoperatoria fue del $7.10 \%$ (1 caso). La estancia hospitalaria media fue de 6.29 días (2-15 días).

El 50\% (7 casos) recibieron adyuvancia. El $71.4 \%$ (10 casos) presentaron recaída de la enfermedad sistémica y solo un paciente tuvo recaída local. La media de tiempo libre de enfermedad fue de 20.6 meses (3-58 meses). La mediana de supervivencia global fue de 30 meses (4-111 meses). La supervivencia fue del $75 \%$ al año, del $55.5 \%$ a los 3 años y del $40 \%$ a los 5 años.

\section{Discusión}

La cirugía de las metástasis suprarrenales ha evolucionado de forma absoluta, pasando de ser un signo de enfermedad diseminada y contraindicación de cirugía a convertirse en una opción terapéutica emergente, segura y con implicaciones pronósticas positivas en determinados tumores y casos seleccionados.

De acuerdo con lo publicado en numerosas series, el tumor que con mayor frecuencia metastatiza en la glándula suprarrenal es el adenocarcinoma pulmonar, alcanzando hasta un $87.5 \% \%^{7}$ de los casos, frente al $42.8 \%$ en nuestra serie. Cuatro pacientes tuvieron metástasis metacrónicas, uno sincrónica y uno antecrónica, siendo esta última muy infrecuente y sin referencia de ningún tratamiento secuencial al respecto ${ }^{8}$.

De la Quintana, et al. ${ }^{9}$ describen el carcinoma pulmonar de células no pequeñas como el más frecuente, con el $51.4 \%$ de los casos, coincidiendo con nuestra serie, en la cual no se encuentra ningún caso de carcinoma microcítico, considerado tradicionalmente una enfermedad extendida y abordable con quimioterapia paliativa ${ }^{4}$. En el momento del 
diagnóstico, tres pacientes presentaban metástasis en otra localización: cerebral, pulmonar y ósea, respectivamente, con buena respuesta a la terapia neoadyuvante. Las indicaciones de neoadyuvancia consideradas en nuestra serie fueron las metástasis en otra localización, antecrónica y sincrónica. Jalón-Monzón, et al. ${ }^{7}$ no consideran la quimioterapia neoadyuvante siempre que el tumor primario esté controlado y no exista enfermedad en otro lugar.

La incidencia de metástasis suprarrenales por carcinoma de células renales se describe en un $2-10 \%$ de los casos, y en las series de autopsias en un $6-29 \%^{3}$. La afectación suprarrenal es rara ${ }^{10}$ y afecta a la glándula homolateral con mayor frecuencia ${ }^{11-13}$. En nuestra serie, un caso tuvo afectación homolateral y dos casos metacrónica. La afectación suprarrenal homolateral puede ocurrir por diseminación hematógena de células aisladas o por trombosis tumoral a través de la vena renal o la vena adrenal, y la extensión directa a través de la cápsula renal puede producirse en tumores de gran tamaño o del polo superior. Se han descrito metástasis metacrónicas hasta 23 años después de una nefrectomía ${ }^{14}$. Lau, et al. $^{15}$, en una serie de 56 casos, observaron 24 lesiones sincrónicas y 32 metacrónicas con un tiempo máximo hasta la aparición de metástasis suprarrenal después de la nefrectomía de 23 años.

Actualmente se considera la vía laparoscópica como el abordaje inicial en el tratamiento quirúrgico de la afectación suprarrenal ${ }^{16}$. En nuestra serie, el abordaje utilizado fue laparoscópico transabdominal y se decidió cirugía abierta en dos casos por tratarse de tumores mayores de $14 \mathrm{~cm}$.

En los pacientes con metástasis únicas en las que es posible su extirpación quirúrgica completa se han referido tasas de supervivencia del $23-35 \%$ a los 5 años ${ }^{13,17,18}$, siendo el pronóstico más favorable en aquellos pacientes con mayor intervalo de tiempo desde la nefrectomía ${ }^{19}$. En nuestra serie, la mediana de supervivencia fue de 30 meses, coincidiendo con la publicada por De la Quintana, et al. ${ }^{9}$, que muestra una mediana de 37,3 meses, y menor que la de la serie de Hatano, et al. ${ }^{6}$, con una media de 63 meses. Cabe mencionar que, en nuestra serie, el $50 \%$ de los pacientes presentaban metástasis en otras localizaciones al momento del diagnóstico.

En nuestra serie, la mortalidad global fue del $7.10 \%$ (1 caso) por metástasis de $15 \mathrm{~cm}$ de hepatocarcinoma, en un paciente cirrótico previamente no diagnosticado.
La supervivencia global al año fue del $75 \%$ en nuestra serie, menor que la de Hatano, et al. ${ }^{6}$, que fue del $92 \%$. A los 5 años es del 40\%, coincidiendo con De la Quintana, et al. ${ }^{9}$, que reportaron un $42,86 \%$, y con Hatano, et al. ${ }^{6}$, que reportaron un 56\%. Jalón-Monzón, et al. ${ }^{7}$ encuentran algunos factores pronósticos asociados a la supervivencia: edad, localización primaria, grado de diferenciación, tipo histológico y tratamiento del primario, pero sin encontrar una correlación significativa. Por otro lado, De la Quintana, et al. ${ }^{9}$ incluyen como factores pronósticos el tamaño $>4 \mathrm{~cm}$, la lateralidad, el intervalo libre de enfermedad, la quimioterapia y la técnica quirúrgica, pero sin encontrar ningún factor que influya en la supervivencia, salvo el tamaño tumoral. Hatano, et al. ${ }^{6}$ describen que la metacronicidad, el tamaño $<4 \mathrm{~cm}$ y el tipo histológico de carcinoma de células renales tienen mejor supervivencia. En nuestra serie no observamos una asociación significativa con ninguno de los factores mencionados.

Las referencias a cirugía de metástasis suprarrenal de origen colorrectal, hepatocarcinoma y carcinoma de mama son, como en nuestra serie, anecdóticas, habiéndose registrado dos casos de origen colorrectal, un caso de hepatocarcinoma y un caso de carcinoma de mama.

En todos nuestros casos, la indicación quirúrgica estaba justificada con fines terapéuticos, previa discusión en el comité de tumores endocrinos.

En los pacientes con carcinoma pulmonar no microcítico y lesiones metastásicas suprarrenales únicas, la cirugía mejora la supervivencia en casos seleccionados: enfermedad primaria controlada, tumor $<4 \mathrm{~cm}$, intervalo libre de enfermedad $>6$ meses y metástasis localizada solo en la glándula suprarrenal ${ }^{8,19}$. Sin embargo, se dispone de escasos datos de pacientes con carcinoma microcítico.

\section{Conclusiones}

No se ha demostrado que en otros tumores diferentes del carcinoma pulmonar no microcítico se mejore la supervivencia global o el intervalo libre de enfermedad. Aun así, en presencia de una masa suprarrenal única, la valoración quirúrgica es mandatoria. Por otro lado, en nuestra serie, el $50 \%$ de los pacientes tenían metástasis localizadas en otros órganos y aun así la supervivencia es similar a la de aquellos pacientes con metástasis única, lo que indica que la cirugía podría ocupar un lugar importante también en este tipo de pacientes, claramente con control de la enfermedad primaria. 


\section{Agradecimientos}

Un enorme agradecimiento al Dr. José María Miguelena, Profesor Titular de Cirugía de la Universidad de Zaragoza, por inculcar y promover el espíritu investigador, y por realizar la revisión y la corrección del presente artículo.

\section{Conflicto de intereses}

Los autores declaran no tener conflictos de intereses.

\section{Responsabilidades éticas}

Protección de personas y animales. Los autores declaran que para esta investigación no se han realizado experimentos en seres humanos ni en animales.

Confidencialidad de los datos. Los autores declaran que han seguido los protocolos de su centro de trabajo sobre la publicación de datos de pacientes.

Derecho a la privacidad y consentimiento informado. Los autores declaran que en este artículo no aparecen datos de pacientes.

\section{Bibliografía}

1. Glomset D. The incidence of metastasis of malignant tumors to the adrenals. Am J Cancer. 1938;32:57-61.
2. Abrams HL, Spiro R, Goldstein N. Metastases in carcinoma; analysis of 1000 autopsied cases. Cancer. 1950;3:74-85.

3. Cingam S, Karanchi H. Cáncer, metástasis suprarrenales. Treasure Island (FL): StatPearls Publishing; 2019.

4. Maciá S, Llorca C, Juárez A, Esquerdo G, Cervera JM, Navío J, et al. Suprarrenalectomía en paciente con metástasis adrenal de carcinoma microcítico de pulmón. Rev Int Grupos Invisto Oncol. 2012;1:32-5.

5. Lam KY, Lo CY. Metastatic tumours of the adrenal glands: a 30-year experience in a teaching hospital. Clin Endocrinol (Oxf). 2002;56:95-101.

6. Hatano K, Horii S, Nakai Y, Nakayama M, Kakimoto KI, Nishimura K. The outcomes of adrenalectomy for solitary adrenal metastasis: a 17year single-center experience. Asia Pac J Clin Oncol. 2020;16:e86-e90.

7. Jalón-Monzón A, Castanedo-Álvarez D, Hevia-Suárez MA, Álvarez-Múgica M, Medina-González A, Escaf-Barmadah S. Resultados de la adrenalectomía en metástasis de cáncer de pulmón. Actas Urol Esp. 2018;42:600-5.

8. Fañanas J, Pérez A, Borrego V, Miguelena J. Suprarrenalectomía antecrónica por metástasis de carcinoma pulmonar. Cir Esp. 2010;87:319-33.

9. De la Quintana A, Martínez G, Arana A, Prieto M, Álvarez I, Martínez L, et al. Cirugía de las metástasis en la glándula suprarrenal: resultados de una serie de 35 pacientes. Cir Esp. 2012;90:634-40.

10. Cruz N, Linares A, Clemente I. Metástasis adrenal metacrónica solitaria contralateral de un carcinoma renal primario: aportación de un nuevo caso. Arch Esp Urol. 2001;54:825-8.

11. Saitoh H, Nakayama M, Nakamura K. Distant metastasis of adenocarcinoma in nephrectomized cases. J Urol. 1982;127:1092-5.

12. Kessler $\mathrm{O}$, Mukamel E, Weinstein R. Metachronous renal cell carcinoma metastasis to the contralateral adrenal gland. Urology. 1998;51:539-43.

13. Von Knobloch, Hegele A, Kälble T. Management of contralateral adrenal metastasis from renal cell carcinoma: possibility of inferior vena cava tumour thrombus. Scand J Urol Nephrol. 2000;34:109-13.

14. Mesorolle B, Mignon F, Travagli J. Late presentation of solitary contralateral adrenal metastasis of renal cell carcinoma. Eur Radiol. 1997;7:557-8.

15. Lau W, Zincke H, Lohse C, Cheville JC, Weaver AL, Blute ML. Contralateral adrenal metastasis of renal cell carcinoma: treatment, outcome, and review. BJU Int. 2003:91:775-9.

16. Elashry O, Claymn R, Soble J. Laparoscopic adrenalectomy for solitary metachronous contralateral adrenal metastasis from renal cell carcinoma. J Urol. 1997:157:1217-22.

17. Sagalowsky A, Molberg K. Solitary metastasis of renal cell carcinoma to the contralateral adrenal gland 22 years after nephrectomy. Urology. 1999;54:162.

18. Sengupta $S$, Leibovich $B$, Blute $M$. Surgery for metastatic renal cell cancer. World J Urol. 2005;23:155-60.

19. Lad T, Piantadosi S, Thomas P, Payne D, Ruckdeschel J, Giaccone G. A prospective randomized trial to determine the benefit of surgical resection of residual disease following response of small cell lung cancer to combination therapy. Chest. 1994;106:320-3. 